\section{Could over-the-counter treatment protect against Parkinson disease?}

Regular doses of ibuprofen could reduce the risk of developing Parkinson disease (PD), according to new research. Ibuprofen-a common and inexpensive over-the-counter drug-could potentially protect the brain's neurons against damage, says lead investigator Xiang Gao, of Harvard Medical School and Harvard School of Public Health, Boston, USA.

\section{4 ...adults who regularly take ibuprofen ... have a lower risk of developing PD compared with nonusers 77}

In a large prospective study, Gao and his colleagues investigated the use of NSAIDs in 136,197 individuals from two cohortsthe Health Professionals Follow-up Study and the Nurses' Health Study. All participants were free of PD at baseline, and were followed up for 6 years. During this time, 291 cases of PD emerged.

Using questionnaires to obtain patients' self-reported analgesic use, the researchers observed a dose-response relationship between the amount of ibuprofen taken per week and the risk of PD. "We found that adults who regularly take ibuprofen - more than twice per week - have approximately one-third lower risk of developing PD compared with nonusers," explains Gao. By contrast, the use of aspirin, acetaminophen or other NSAIDs was not significantly linked to the risk of developing PD in the future.

The prospective study design and high follow-up rate involved in Gao et al.'s study meant that bias from selection and recall could be avoided. Gao's team conducted a meta-analysis using all available material previously published on similar research to confirm their results.

The neuroprotective properties of ibuprofen, compared with other NSAIDs, have already been demonstrated in dementia-its long term use has been associated with a reduced risk of Alzheimer disease by lowering levels of amyloid- $\beta_{42}$, which is linked to the pathogenesis of the disease.

Although neuroinflammation sometimes offers beneficial effects, a substantial degree of inflammation can lead to the degeneration of dopaminergic

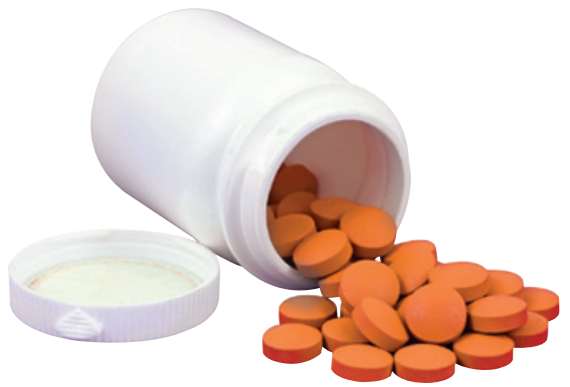

neurons. A generic anti-inflammatory effect is an unlikely explanation for the reduced risk of $\mathrm{PD}$, however, since aspirin or other commonly used analgesics do not show similar effects.

The mechanism through which ibuprofen exerts its neuroprotective effects will require further research. One explanation that might be explored is that ibuprofen acts a ligand for peroxisome proliferator-activated receptor- $\gamma-\mathrm{a}$ known therapeutic target for PD.

Gao adds that further clinical trials are now needed to determine whether ibuprofen could be involved in slowing the rate of progression of $\mathrm{PD}$ in patients who are in the early stages of the disease.

Carolyn McSharry

Original article Gao, X. et al. Use of ibuprofen and risk of Parkinson disease. Neurology 76, 863-869 (2011) 